\title{
Introduction: High-Temperature Corrosion in Energy-Related Systems
}

\author{
Kazuya Kurokawa ${ }^{1}$ - Masayuki Yoshiba ${ }^{2}$. \\ Shigenari Hayashi ${ }^{3}$
}

Published online: 15 March 2016

(C) Springer Science+Business Media New York 2016

Materials used at elevated temperatures are required to have both excellent mechanical properties and oxidation/corrosion resistance. These tend to be opposing properties from the standpoint of alloying, which presents technical challenges to the extent that there is an ever-increasing need to understand better the fundamental issues affecting optimal design of high-temperature materials. Indeed, recent demands for improved energy savings, reduced environmental pollution, and decreased emissions of $\mathrm{CO}_{2}$ and other greenhouse gases impose the need for further advancements in high-temperature materials.

A series of specialized meetings entitled, International Symposium on HighTemperature Oxidation and Corrosion (ISHOC), have been held regularly in Japan since 2000. The fourth of these meetings was ISHOC-2014, which was held in the northern city of Hakodate on July 23-27, 2014. The main objective of the ISHOC series is to address current issues of high-temperature materials used in harsh service environments. This includes providing further elucidation of fundamental

Kazuya Kurokawa

kurokawa@tomakomai-ct.ac.jp

Masayuki Yoshiba

yoshiba@tmu.ac.jp

Shigenari Hayashi

s.hayashi@mtl.titech.ac.jp

1 National Institute of Technology, Tomakomai College, 443 Nishikioka, Tomakomai 059-1275, Japan

2 Faculty of Urban Liberal Arts, School of Science and Engineering, Division of Engineering, Tokyo Metropolitan University, 1-1 Minami-Osawa, Hachioji-Shi, Tokyo 192-0397, Japan

3 Department of Metallurgy and Ceramics Science, Graduate School of Science and Engineering, Tokyo Institute of Technology, 2-12-1-S8-3, Ookayama, Meguro-Ku, Tokyo 152-8552, Japan 
mechanisms governing a particular mode of degradation and guiding technical solutions for arriving at improved materials. At ISHOC-2014, there was participation by a total of 154 scientists. Of that number, 92 were from Japan and 62 were from 15 different countries. A number of attendees were encouraged to submit a paper based on their presentation for publication in one of two topical special issues of Oxidation of Metals:

I. Processes and performance in harsh environments

II. High-temperature corrosion in energy-related systems.

The February 2016 issue of Oxidation of Metals presented papers on Topic I, while this current issue presents papers on Topic II. Similar to the Topic I issue, the papers in this Topic II issue are by leading researchers in the field of hightemperature corrosion. We are confident that these papers will serve as a great resource in years to come. 